\title{
Autenticidade e preservação de Registros Eletrônicos em Saúde: proposta de modelagem da cadeia de custódia das informações orgânicas do Sistema Único de Saúde
}

\author{
Authenticity and preservation of Electronic Records in Health: proposal of \\ chain of custody model for the Unified Health System organic information
}

\author{
Autenticidad y preservación de Registros Electrónicos en de Salud: \\ propuesta de modelado de la cadena de custodia de las informaciones \\ orgánicas del Sistema Único de Salud
}

Rodrigo França Meirelles ${ }^{1, a}$

rodrigomei@ufba.br | https://orcid.org/o0oo-0001-5535-7208

Francisco José Aragão Pedroza Cunha ${ }^{1, b}$

pedrozaici@ufba.br | https://orcid.org/oooo-0003-2770-7818

\footnotetext{
${ }^{1}$ Universidade Federal da Bahia, Instituto de Ciência da Informação. Salvador, BA, Brasil.

a Mestrado em Ciência da Informação pela Universidade Federal da Bahia.

${ }^{\text {b }}$ Doutorado em Difusão do Conhecimento pela Universidade Federal da Bahia.
}

\section{RESUMO}

Este artigo apresenta uma proposta de modelagem conceitual da cadeia de custódia para os Registros Eletrônicos em Saúde (RES). O interesse em conhecer os critérios de autenticidade dos RES encaminhados dos serviços até o Portal do DATASUS gerou esta investigação. A pesquisa é exploratória e descritiva por meio de uma revisão sistemática da literatura e documental para prospecção e análise sobre autenticidade e preservação dos RES. A partir dos resultados encontrados foram identificados os custodiadores e a proposta de modelagem da cadeia de custódia para o Sistema Único de Saúde (SUS). Espera-se que os custodiadores modelados assimilem subsídios para a criação de repositórios digitais em saúde confiáveis e recomenda-se a adoção e a incorporação dos processos de gestão arquivística de documentos articulados com os da TI.

Palavras-chave: Registros Eletrônicos em Saúde; Preservação digital; Autenticidade; Repositórios arquivísticos; Cadeia de custódia. 


\section{ABSTRACT}

This article presents a proposal of conceptual modeling of the chain of custody for Electronic Health Records (EHR). The curiosity to know the authenticity criteria of the EHR sent from the services to the DATASUS Portal generated this investigation. The research is both exploratory and descriptive by means of a systemic literature and documental review for the prospection and analysis on authenticity and preservation of the EHR. According to the results found, the custodians and the proposal of custody chain modeling for Unified Health System (SUS) were identified. The modeled custodians are expected to assimilate subsidies for the creation of reliable digital health repositories. It is recommended that they adopt and incorporate the articulated processes of archival management to those from the IT.

Keywords: Electronic Health Records; Digital preservation; Authenticity; Archival repositories; Chain of custody.

\section{RESUMEN}

Este artículo presenta una propuesta de modelado conceptual de la cadena de custodia para Registros Electrónicos en Salud (RES). La curiosidad por conocer los criterios de autenticidad de los RES enviados desde los servicios hacia el Portal DATASUS generó esta investigación. La investigación es exploratoria y descriptiva a través de una revisión sistemática de literatura y documental para la prospección y análisis de autenticidad y preservación de los RES. A partir de los resultados encontrados, se identificaron los custodios y la propuesta de modelado de la cadena de custodia para el Sistema Único de Salud (SUS). Se espera que los custodios modelados asimilen los subsidios para la creación de repositorios digitales en salud confiables y se recomienda la adopción e incorporación de los procesos de gestión de archivos de documentos articulados con los de TI.

Palabras clave: Registros Electrónicos en Salud; Preservación digital; Autenticidad; Repositorios de archivos; Cadena de custodia.

Este artigo compõe o Dossiê Preservação Digital.

Contribuição dos autores:

Concepção e desenho do estudo: Rodrigo França Meirelles, Francisco José Aragão Pedroza Cunha.

Aquisição, análise ou interpretação dos dados: Rodrigo França Meirelles, Francisco José Aragão Pedroza Cunha.

Redação do manuscrito: Rodrigo França Meirelles, Francisco José Aragão Pedroza Cunha.

Revisão crítica do conteúdo intelectual: Rodrigo França Meirelles, Francisco José Aragão Pedroza Cunha.

Declaração de conflito de interesses: não há.

Fontes de financiamento: Edital Universal do CNPq de 2016.

Considerações éticas: Aprovado pelo Comitê de Ética em Pesquisa da Escola de Enfermagem da UFBA. Parecer: 3.509.198.

Agradecimentos/Contribuições: não há.

Histórico do artigo: submetido: 18 maio 2020 | aceito: 05 ago. 2020 | publicado: 30 set. 2020.

Apresentação anterior: não houve.

Licença CC BY-NC atribuição não comercial. Com essa licença é permitido acessar, baixar (download), copiar, imprimir, compartilhar, reutilizar e distribuir os artigos, desde que para uso não comercial e com a citação da fonte, conferindo os devidos créditos de autoria e menção à Reciis. Nesses casos, nenhuma permissão é necessária por parte dos autores ou dos editores. 


\section{INTRODUÇÃO}

Os constantes avanços tecnológicos e o acesso à internet impulsionaram a produção e o uso de informações e, consequentemente, de documentos em suporte digital, em volume e diversidade. O uso dos documentos digitais não diminuiu a relevância dos analógicos, porém o aumento da produção em meio digital afetou o modo como instituições tradicionais de documentação (e.g. arquivos, bibliotecas, centros de documentação e museus), bem como as organizações dos primeiro, segundo e terceiro setores (e.g. públicas, privadas e sem fins lucrativos e/ou ministérios de governos, bancos, hospitais, instituições de ensino superior, escolas), passam a gerir os acervos informacionais ${ }^{1}$.

As informações em saúde subsidiam as tomadas de decisão dos profissionais e gestores ${ }^{2}$ para as ações de atenção, controle e participação social no Brasil. Pinto, Freitas e Figueiredo ${ }^{3}$ salientam que o "uso de Sistemas de Informações em Saúde para apoio à gestão micro e macro tem sido (...) estratégia de Estado em todos os níveis dos sistemas de saúde (...). [Superar os] desafios de integração entre os mais diversos subsistemas (...) significa proporcionar uma melhor alocação dos recursos na saúde e redução do desperdício”. Cabe lembrar que a informação em saúde é “(...) de natureza muito particular, e não se prende unicamente às questões referentes ao domínio da terapêutica médica, porém ao registro de todas as ações efetivadas por outros profissionais que se inserem nessa área, além daquelas que contribuem direta ou indiretamente para a qualidade no atendimento aos pacientes. (...) diz respeito a todos os problemas que o setor de saúde enfrenta para a manutenção da normalidade referente ao estado da pessoa doente e contribui para o desenvolvimento de políticas públicas de informação no contexto da saúde. (...) esse tipo de informação contempla questões que dizem respeito às patologias (per-si), à saúde, à legislação, à gestão, à padronização, à nutrição, às condições socioeconômicas, ao credo, à educação, às tecnologias, à terminologia, além de outras"4.

As informações geradas nos Organismos Produtores de Serviços de Atenção à Saúde (OPSAS) são registradas em suportes analógicos ou eletrônicos configurando tecnologias (e.g. documentos, arquivos, sistemas de informação e repositórios). Tais tecnologias são entendidas como mecanismos de difusão de conhecimentos entre os serviços, sistemas e redes de atenção e inovação à saúde ${ }^{5}$ e dependentes de registros informacionais autênticos e confiáveis. Para tanto, a adoção e a assimilação de tecnologias avançadas de gestão e a preservação de documentos entre os OPSAS (e.g. de hospitais) ${ }^{6}$ são necessárias.

No Brasil, o Sistema Único de Saúde (SUS) e o Sistema de Saúde Suplementar conformam o sistema de saúde brasileiro. O SUS é essencial para saúde pública e, justamente pela sua amplitude, é objeto de estudo de diversas áreas do conhecimento. O Departamento de Informática do SUS (DATASUS) foi criado com o objetivo de informatização das atividades do SUS e tem como missão fornecer sistemas de informação e suporte de informática. O DATASUS é o responsável pela gestão dos Sistemas de Informação em Saúde (SIS) e dos sistemas internos de gestão do Ministério da Saúde ${ }^{3,7}$.

No tocante à identificação, classificação, avaliação, preservação, descrição e difusão das informações em saúde, o campo da Arquivologia vem ampliando seus estudos relacionados à Gestão Arquivística de Documentos (GAD) e aos repositórios institucionais, os quais contribuem diretamente para a gestão e inovação do SUS. No Brasil, o Conselho Nacional de Arquivos (CONARQ) disponibiliza uma série de documentos e recomendações referentes à GAD.

A GAD, os Registros Eletrônicos em Saúde (RES), os sistemas de arquivos e os repositórios institucionais são explicitamente citados nas diretrizes preconizadas na Política Nacional de Informação e Informática em Saúde (PNIIS). Os RES são entendidos como "repositório de informações processáveis sobre o cuidado em saúde do indivíduo, armazenadas e transmitidas de forma segura e acessível por múltiplos usuários autorizados”2. A Informação e Tecnologia de Informação em Saúde (ITIS) para o desenvolvimento do SUS é também apresentada no Plano Diretor de Desenvolvimento da Informação e Tecnologias de Informação em Saúde 
(PlaDITIS). No $2^{\circ}$ PlaDITIS é suscitado o debate sobre a necessidade do acompanhamento do uso da ITIS a serviço da sociedade para garantir atenção integral à saúde, com qualidade equanimemente distribuída ${ }^{8}$.

Alinhadas às diretrizes e aos princípios do SUS (e.g. universalidade, equidade, integralidade, participação popular e controle social, descentralização-político-administrativa, hierarquização e regionalização dos serviços) e à política de governo eletrônico, no documento Estratégia e-Saúde para o Brasil, são delineadas ações para o uso da ITIS, visando alcançar os objetivos do Plano Nacional de Saúde (PNS) até o ano de 20209. O interesse em conhecer os critérios de autenticidade dos RES, encaminhados dos OPSAS até os bancos de dados do Portal do DATASUS, e relacioná-los às diretrizes acerca da cadeia de custódia desses registros - disponibilizados nesses bancos de dados - gerou a questão: como os agentes custodiadores do SUS podem garantir a autenticidade e a preservação dos RES?

A cadeia de custódia é compreendida como a "linha contínua de custodiadores de documentos arquivísticos (desde o seu produtor até o seu legítimo sucessor) pela qual se assegura que esses documentos são os mesmos desde o início, não sofreram nenhum processo de alteração e, portanto, são autênticos" ${ }^{\prime 10}$. Já as informações orgânicas são o "produto das atividades executadas na organização, elas são produzidas no contexto do exercício das funções administrativas, o registro físico das transações de uma determinada atividade, tarefa ou tomada de decisão"11.

Nesse sentido, os documentos arquivísticos em saúde são constituídos de informações orgânicas geradas nos serviços assistenciais e registradas em um determinado suporte (e.g. analógico ou eletrônico). Assim, os RES conformam os documentos arquivísticos em saúde e o objetivo desta investigação é o de apresentar uma proposta de modelagem conceitual da cadeia de custódia para o SUS (i.e. das informações orgânicas em saúde).

Faz-se necessária a difusão, adoção e a assimilação entre os agentes de saúde e as organizações/ instituições, respectivamente, da GAD, de sistemas de arquivos e de repositórios arquivísticos. Tais conhecimentos, além de proporcionar melhorias na prestação dos serviços de atenção à saúde para o SUS, subsidiam a criação de Repositórios Digitais Confiáveis (RDC-Arq) em saúde.

\section{METODOLOGIA}

A pesquisa tem caráter interdisciplinar por articular múltiplas áreas do conhecimento (e.g. Administração, Arquivologia, Biblioteconomia, Comunicação, Computação, Ciências Cognitivas, Ciência da Informação, Difusão do Conhecimento e Saúde Coletiva). Essa articulação possibilita inferir sobre a GAD (e.g. critérios de autenticidade e preservação) e a cadeia de custódia dos RES (e.g. modelo OAIS e (RDC-Arq), a partir da representação e da difusão do conhecimento por meio de mecanismos e estruturas de transferência de informações orgânicas nos sistemas e nas redes de atenção à saúde. Tais sistemas e redes, nesta pesquisa, são compreendidos pelos OPSAS e pelo Portal do DATASUS, os quais geram, recebem e acumulam documentos arquivísticos diariamente.

Em relação ao objetivo, trata-se de uma pesquisa exploratória, por ser realizada em uma área na qual há pouco conhecimento acumulado e sistematizado sobre a modelagem conceitual do fenômeno (i.e. a infraestrutura de sistemas de gestão da preservação das informações e (RDC-Arq) $\Leftrightarrow$ apresentando os agentes custodiadores dos RES no âmbito das Redes de Atenção à Saúde (RAS) do SUS). Ao mesmo tempo, esta pesquisa é também descritiva, por pontuar ferramentas, padrões e melhores práticas sobre (RDC-Arq) (i.e. requisitos e critérios para a autenticidade e preservação dos RES e manutenção da cadeia de custódia).

Para o levantamento das informações da revisão de literatura e documental, foram utilizados os elementos da técnica de Revisão Sistemática (RS) para a prospecção e análise dos requisitos, critérios e das características para os Repositórios Arquivísticos Digitais Confiáveis em saúde de modo mais abrangente e imparcial. Para a criação da proposta de modelagem conceitual da cadeia de custódia do SUS foram 
utilizados como custodiares os OPSAS, com termo de adesão a uma rede de inovação e aprendizagem em gestão hospitalar, vinculados ao SUS, e o Portal do DATASUS.

A RS consiste em uma revisão planejada, que utiliza métodos sistemáticos para identificar, selecionar e avaliar estudos relevantes sobre um determinado objeto/fenômeno/assunto t2 $^{12}$. A RS requer o estabelecimento de uma pergunta clara; de uma estratégia de busca; de critérios de inclusão e exclusão dos artigos; de uma análise criteriosa da qualidade da literatura selecionada ${ }^{13}$.

A prospecção foi realizada com base em artigos científicos, nos idioma português, inglês, francês e espanhol, tendo como fonte de pesquisa as bases de dados Scopus, Web of Science e Google. As bases foram selecionadas em razão da sua representatividade nas áreas do estudo e relevância na comunidade científica. A coleta foi ampliada para pesquisas no Google para compor a revisão documental, já que, em geral, Leis, Resoluções, Regulamentações, Políticas, Normas, Tutorias e demais documentos não são localizados na íntegra em bases de dados científicas.

Os seguintes descritores foram usados como estratégias de busca para o levantamento de artigos científicos: "Sistemas de Informação em Saúde (SIS)", "padrões de representação da informação e do conhecimento", "preservação e autenticidade de documentos digitais", "Portal do DATASUS", "Sistema Informatizado de Gestão Arquivística de Documentos (SIGAD)", "Repositórios Arquivísticos Digitais Confiáveis (RDC-Arq)" e "modelo Open Archival Information System (OAIS)" associados aos descritores "saúde", "SUS" e "DATASUS". Nas bases de dados foram utilizados os operadores lógicos booleanos e demais ferramentas de refinamento de busca disponíveis nas bases.

Foram selecionados, incialmente, 283 textos - após leitura de títulos, resumo e palavras-chave, introdução e conclusão/considerações finais -, inseridos e organizados no software Mendeley para facilitar o gerenciamento e acesso. Em uma segunda etapa, foram lidos 118 textos em sua integralidade, estabelecendo como critério de inclusão e exclusão a análise criteriosa da qualidade da literatura selecionada, conforme recomendado por Sampaio e Mancini ${ }^{13}$, como elementos de uma RS, sendo utilizados, ao fim do percurso, 39 textos que possuem pesquisas mais relevantes sobre a temática tratada neste artigo.

\section{AUTENTICIDADE E PRESERVAÇÃO DIGITAL DOS REGISTROS ELETRÔNICOS EM SAÚDE (RES)}

A preservação digital organizacional é dependente de princípios, procedimentos e técnicas arquivísticas que subsidiem dispositivos para a autenticidade dos registros eletrônicos (e.g. políticas, planos, critérios, requisitos, sistemas de preservação, entre outros). As políticas regulamentam um conjunto de processos, estratégias e ferramentas (i.e. tecnologias, formatos, hardware, software e suportes técnicos disponíveis) para armazenar e recuperar registros gerados e recebidos em razão das atividades organizacionais.

Em geral, os sistemas de preservação dos registros das informações asseguram o armazenamento, o acesso e a interoperabilidade dos dados e detêm requisitos capazes do gerenciamento da autenticidade e da manutenção do histórico de alterações nos registros, proporcionando, dessa forma, uma integridade efetiva ${ }^{14}$. Uma política de preservação digital prevê uma atualização contínua, em razão da obsolescência tecnológica, de acordo com as demandas. Ela também necessita da implantação de um planejamento com base em diagnóstico do acervo institucional. Alguns estudos revelam que as políticas de preservação de registros das atividades exigem requisitos minuciosos a serem contemplados e acabam sendo descontinuadas, ou nem mesmo incorporadas, por muitas das instituições ${ }^{15,16}$.

O planejamento de uma política dessa natureza necessita considerar a infraestrutura adequada e equipe multidisciplinar com profissionais das áreas administrativa, arquivística, informática e das áreas de concentração do acervo (i.e. da atividade-fim da instituição). É recorrente a equipe se qualificar permanentemente sobre as tendências e evoluções no campo da preservação digital. Bountouri, Gratz e 
Sanmartin ${ }^{17}$ recomendam, além dos procedimentos técnicos de rotina, algumas ações: a participação em conferências científicas, em comitês científicos da disciplina relacionada, em seminários, como a Conferência Internacional sobre Preservação Digital e o Grupo de Trabalho Preservation Metadata: Implementation Strategies (PREMIS); o estabelecimento de um contrato de consultoria com empresa especializada; além da divulgação de listas de eventos, de notícias sobre o tema, entre outros.

O PREMIS é uma iniciativa importante junto à comunidade de preservação digital (e.g. PREMIS Data Dictionary for Preservation Metadata). É um documento de referência que apresenta um conjunto de elementos de metadados de preservação para apoiar sistemas que gerenciam objetos digitais. Outras iniciativas de destaque são as desenvolvidas pelos grupos de trabalho liderados pelo Research Library Group (RLG) e pelo Online Computer Library Center (OCLC). Nesse contexto, destaca-se também a Trustworthy Repositories Audit \& Certification: Criteria and Checklist (TRAC), base para elaboração da norma ISO 16363:2012 e para os Repositórios Digitais Confiáveis.

No Brasil, o CONARQ desenvolveu atividades e gerou documentos e resoluções norteadores (e.g. Carta para a Preservação do Patrimônio Arquivístico Digital do CONARQ de 2004) ${ }^{18}$. Nesta carta é ressaltada a importância das políticas, dos procedimentos e sistemas de preservação que originou as Diretrizes para a Implementação de Repositórios Arquivísticos Digitais Confiáveis (RDC-Arq) ${ }^{19}$. Outra iniciativa de relevância é a do Instituto Brasileiro de Informação em Ciência e Tecnologia (Ibict) que fomenta e promove serviços de preservação digital de documentos eletrônicos brasileiros - a Rede Cariniana.

As políticas de preservação digital têm um papel fundamental nas instituições para o planejamento e a gestão arquivística de documentos. Essas políticas são compreendidas como dispositivos que asseguram a autenticidade dos documentos em longo prazo $^{20}$. É recomendável que os processos de implantação e de avaliação das políticas se baseiem em diretrizes e requisitos internacionalmente credíveis com a aplicação de estratégias e ferramentas de preservação digital.

No Ministério da Saúde, os assuntos relativos às políticas de preservação e segurança da informação em saúde são de responsabilidade do DATASUS 9 . O Portal do DATASUS informa que todos os sistemas e aplicativos utilizados, no âmbito do SUS, seguem normas de referência internacional, a saber: ISO 27001 - Sistemas de gestão de segurança da informação - Requisitos; ISO 27002 - Código de prática para controles de segurança da informação; ISO 27003 - Sistemas de gestão da segurança da informação - Orientações; ISO 27004 - Sistemas de gestão da segurança da informação - Monitoramento, medição, análise e avaliação; ISO 27005 - Gestão de riscos de segurança da informação9.

Se por um lado, o uso de critérios e requisitos preconizados pelas normas e políticas de preservação é fundamental para subsidiar a garantia da integridade e identidade dos registros, da segurança da informação e das comunicações institucionais. Por outro, a prática da implantação de uma política de preservação dos RES requer cuidados específicos com documentos que garantam a autenticidade e a cadeia de custódia por meio de princípios, procedimentos, normas e técnicas arquivísticas aplicados ao ciclo de vida dos documentos ${ }^{21}$.

É recomendado que o gerenciamento dos documentos de um Repositório Digital Confiável esteja de acordo com o modelo de referência OAIS - modelo conceitual desenvolvido pelo Consultative Committee for Space Data Systems (CCSDS), que resultou na norma ISO 14721:200328. O OAIS estabelece a formação de pacotes de informação envolvendo os documentos digitais (i.e. informação de conteúdo) e seus metadados (i.e. informação de representação). Este modelo e outros documentos dessa natureza auxiliam a implantação de uma política de preservação digital no âmbito dos OPSAS, a saber: a) Diretrizes para a presunção de autenticidade de documentos arquivísticos digitais; b) Diretrizes para a implementação de Repositórios Arquivísticos Digitais Confiáveis; c) Plano Diretor de Desenvolvimento da Informação e Tecnologias de Informação em Saúde (PlaDITIS); d) Política Nacional de Informação e Informática em Saúde (PNIIS); e) 
Modelo de Requisitos para Sistemas Informatizados de Gestão Arquivística de Documentos (e-ARQ Brasil); e f) Orientação Técnica ${ }^{\circ} 3$ do CONARQ - Cenário de uso dos RDC-Arq, em conjunto com um Sistema Informatizado de Gestão Arquivística de Documentos (SIGAD) ${ }^{21}$.

É recomendável que sistemas de informação estejam em conformidade com padrões que permitam a interoperabilidade com sistemas informatizados de documentos arquivísticos, entre os quais destaca-se o Open Archives Initiative Protocol Metadata Harvesting (OAI-PMH). O OAI-PMH, utilizado com sucesso no modelo de acesso livre à informação científica, caracteriza-se como uma solução tecnológica para coleta de registros de metadados em repositórios digitais e estabelece a comunicação entre provedores de dados e serviços. Outros padrões e codificações para metadados são relevantes, a saber: a) codificação de metadados descritivos, administrativos e estruturais - Metadata Encoding and Transmission Standard (METS); b) Encoded Archival Description (EAD); c) codificação de metadados descritivos de documentos arquivísticos; e d) Padrões de Interoperabilidade de Governo Eletrônico (ePING), para os órgãos e as entidades do Governo Federal ${ }^{19}$. Este último, o ePING, consta como padrão utilizado pelo DATASUS e é recomendado no Estratégia e-Saúde para o Brasil (2014) para proporcionar a integração das Redes de Atenção à Saúde (RAS) e para um efetivo Sistema Nacional de Informação em Saúde (SNIS).

O DATASUS, em razão da situação de enfrentamento à pandemia, criou um Plano de Contingência estabelecendo estratégias para as Tecnologias da Informação e Comunicação (TIC) e um Comitê de Gestão de Crise do Novo Coronavírus (Covid-19) para avaliar e propor melhorias na gestão dos SIS. O Plano de Contingência prevê a integração das notificações e a vigilância epidemiológica junto à Rede Nacional de Dados em Saúde (RNDS). A RNDS tem como objetivo integrar e disseminar as informações de saúde no país para proporcionar mais celeridade e gestão integrada das informações ${ }^{22}$. Contudo, diretrizes relacionadas à cadeia de custódia dos RES não são evidenciadas na plataforma RNDS.

O custodiador é o agente responsável pela custódia física e legal e pela preservação dos documentos (i.e. das informações orgânicas); pela proteção e garantia do acesso contínuo às cópias autênticas de registros inativos do criador ou produtor ${ }^{23}$. Tal condição é contemplada com o uso do modelo referência OAIS, em conformidade com os principais sistemas e listas de verificação de processos de auditoria e certificação internacionais ${ }^{24}$.

As auditorias dessa natureza têm por objetivo a verificação e avaliação das metodologias que a instituição adota, ou seja, elas observam se essas metodologias estão em conformidade com as normas internacionais relacionadas às políticas de preservação. As auditorias também têm como atribuição a aferição do comprometimento das ações, em relação ao que foi planejado nas políticas sobre a infraestrutura e os procedimentos técnicos, permitindo avaliar a segurança do repositório digital ${ }^{15,25}$. Por meio dos requisitos de avaliação é estabelecido o grau de confiabilidade do repositório digital, concedendo ou não a certificação.

As auditorias podem ter custos elevados para determinadas organizações, sendo então necessário conhecer e avaliar quais as melhores opções, de acordo com as características de cada repositório. As diversas auditórias contribuem de modos distintos por possuírem características específicas podendo assim ser utilizadas em conjunto para realizar as avaliações e possuir as certificações necessárias. Esta investigação não tem como objetivo detalhar o funcionamento das certificações existentes, mas salientar a importância de seu uso para avaliação dos repositórios e destacar a necessidade de profissionais qualificados para a implementação, para o monitoramento e avaliação dos requisitos de autenticidade junto aos custodiadores dos RES no âmbito do SUS (e.g. dos OPSAS, da Atenção Primária à Saúde [APS], da atenção especializada e da atenção de alta complexidade).

O aumento da produção de documentos arquivísticos em formato digital, tanto os nato-digitais quanto os digitalizados (i.e. documentos produzidos em suporte papel e que são digitalizados por scanner e armazenados em suporte eletrônico), tem gerado desafios para a preservação. Entre os desafios encontra- 
se o de garantir a autenticidade e o acesso contínuo aos conteúdos e à forma dos documentos frente aos constantes avanços tecnológicos em conformidade com a legislação brasileira.

A Constituição brasileira de $1988^{26}$ assegura a proteção à intimidade das informações registradas e, recentemente, a Lei ${ }^{0}$ 13.709/2018, intitulada Lei Geral de Proteção de Dados Pessoais (LGPDP) ${ }^{27}$, em seu art. $5^{\circ}, \mathrm{I}$, considera qualquer informação relacionada a uma pessoa identificada ou identificável como um dado pessoal sensível. A Lei de Acesso à Informação (LAI), de $\mathrm{n}^{0}{ }^{12.527} / 2011^{28}$, regulamenta o direito constitucional de acesso às informações. No âmbito da saúde, o Código de Ética Médica ${ }^{29}$, do Conselho Federal de Medicina, versa sobre questões éticas relacionadas ao acesso e uso das informações em saúde. Neste sentido, o custodiador dos documentos arquivísticos em saúde exerce a atribuição de garantir a confiabilidade desses documentos por meio de condutas éticas amparadas na legislação vigente.

Se por um lado, a ética assistencial converge para as dimensões da privacidade e da confidencialidade das informações em saúde; por outro, faz-se necessário distinguir a "ética normativa" da consciência ética, própria e apropriada de cada ser humano ${ }^{30}$. O OAIS, por exemplo, quando é tomado de modo literal, ou interpretado superficialmente, pode não atender ao que propõe e pode não alcançar uma estratégia de preservação mais robusta ${ }^{24}$. Nesse sentido, não é recomendado aplicar políticas, regulamentações e modelos de referência sem pensar nas práticas de cada indivíduo e de acordo com a realidade de cada organização por meio do emprego não apenas de tecnologias, mas, também, de qualificações, debates e diálogos entre os envolvidos para que as condutas sejam assimiladas e efetivadas com uma consciência ética própria e apropriada.

Esses cuidados em relação à adoção das políticas de quaisquer naturezas e, em particular, as de informação, devem começar bem antes de os custodiadores tomarem medidas legais de custódia física dos registros para garantir a autenticidade, confiabilidade e precisão por meio de diretrizes específicas de uso, a partir da GAD ${ }^{31}$. Nessa perspectiva, realizar uma reflexão de como são gerados e mantidos os RES estimula a criação de um ambiente colaborativo, que é essencial para preservação, em longo prazo, observando a execução e avaliação das atividades de modo efetivo ${ }^{31}$.

A presunção da autenticidade dos documentos arquivísticos digitais garante a evidência de identidade e integridade do documento (i.e. componentes da autenticidade) ou ao menos minimiza os riscos ${ }^{10}$. Nos documentos arquivísticos digitais, a identidade é relacionada aos atributos (i.e. os metadados) associados ao documento; e a integridade, à forma fixa (i.e. apresentando a mesma forma de quando foi armazenado) e ao conteúdo estável (i.e. permanecendo completo e inalterável) ${ }^{32}$. As características de fixidez são codificadas como parte dos metadados de proveniência (i.e. do agente que gerou ou criou o registro) para auxiliar na comprovação da autenticidade de um documento digital ao longo do tempo ${ }^{17}$.

Os metadados de proveniência são uma das etapas mais significativas no ciclo de vida de um documento digital, pois registram a custódia de todas as atividades que possam ter sido produzidas e identificam qualquer tipo de mudança durante esse ciclo ${ }^{17}$. Para tanto, é necessário que as políticas e os procedimentos implementados pela organização garantam que todas as atividades realizadas pelos produtores, custodiadores e demais profissionais envolvidos sejam monitoradas para assegurar a autenticidade dos RES.

É recomendável que as técnicas de autenticação estejam apoiadas em políticas arquivísticas: "A autenticação é a declaração da autenticidade feita em um dado momento por uma pessoa autorizada para tal. Enquanto declaração, a autenticação não garante necessariamente a autenticidade do documento, na medida em que se pode declarar como autêntico algo que não é. Da mesma forma, um documento pode ser considerado autêntico sem que nele conste uma autenticação" ${ }^{10}$. No Brasil, a Medida Provisória ${ }^{0}$ 2.200-2/2001 estabeleceu a Infraestrutura de Chaves Públicas Brasileira (ICP-Brasil) ${ }^{33}$ como responsável para viabilizar a emissão dos certificados digitais e garantir a autenticidade e a validade jurídica dos documentos.

O modelo adotado pelo Brasil foi o de certificação com raiz única, Autoridade Certificadora Raiz (ACRaiz), que tem o papel de credenciar e descredenciar os demais participantes da cadeia de custódia de 
documentos digitais, supervisionar e fazer auditoria dos processos das Autoridades Certificadoras (AC) da ICP-Brasil, Autoridades de Carimbo do Tempo (ACT) da ICP-Brasil, Prestadores de Serviço Biométrico (PSBio), Prestadores de Serviço de Confiança (PSC), Prestadores de Serviço e Suporte (PSS), Autoridades de Registro (AR) da ICP-Brasil.

As AC são "entidades, públicas ou privadas, subordinadas ao ICP-Brasil, responsáveis por emitir, distribuir, renovar, revogar e gerenciar certificados digitais" ${ }^{33}$. As assinaturas são realizadas por meio de aplicativos que registram os dados das pessoas autorizadas e são validadas por senha e por dispositivos de segurança, como token, e-mail, SMS, chave simétrica, biometria, pergunta-chave, identidade digital, entre outros, para a realização das assinaturas digitais, tanto na esfera pública quanto na privada ${ }^{33}$.

A adoção de sistemas de certificação digital para operações dos SIS do SUS é um procedimento recorrente para garantir a segurança das informações prestadas e atender a Lei Complementar de $\mathrm{n}^{0} 141$, de 13 de janeiro de 2012 (LC 141/2012) sobre os dados das despesas com ações e serviços públicos de saúde ${ }^{33,34}$. Vale registrar que os dados inventariados sobre os cuidados da atenção à saúde de uma pessoa estão em uma tipologia documental denominada de Prontuário do Paciente (PP).

O PP é um “(...) documento único constituído de um conjunto de informações, sinais e imagens registradas, geradas a partir de fatos, acontecimentos e situações sobre a saúde do paciente e a assistência a ele prestada, de caráter legal, sigiloso e científico, que possibilita a comunicação entre membros da equipe multiprofissional e a continuidade da assistência prestada ao indivíduo"35. Em relação ao PP, no manual dos sistemas do Prontuário Eletrônico do Cidadão (PEC), para o Programa de Informatização das Unidades Básicas de Saúde (PIUBS) é recomendado que os sistemas de PEC estejam em conformidade com o Nível de Garantia de Segurança 1 (NGS1) ${ }^{34}$.

Os gestores do SUS (i.e. nos níveis municipal, distrital, estadual e federal), dos sistemas privados e do setor de saúde suplementar, são responsáveis por providenciar a infraestrutura para a implantação do PEC, segundo os manuais do sistema e a legislação vigente ${ }^{34}$. É necessária, portanto, a viabilização dos recursos alocados especificamente para a implantação do PEC. Caso contrário, a sua adoção fica inviabilizada em muitos municípios, por falta de infraestrutura adequada, o que compromete a garantia dos registros em uma cadeia de custódia.

Outra tecnologia que está em vigência para a autenticidade de registros digitais é a blockchain utilizada em diversas aplicações, e que ganhou destaque por ser a responsável na garantia da segurança das transações financeiras da criptomoeda bitcoin (i.e. moeda digital). A blockchain foi definida no código fonte original da bitcoin, criada em 2008 e disponibilizada em código aberto em 2009, após publicação do artigo de seu criador, Satoshi Nakamoto. Esta tecnologia possui uma estrutura de dados que permite que transações sejam realizadas garantindo a autenticidade do registro, por meio de uma infraestrutura dividida em diversos blocos, que juntos formam uma cadeia de blocos, conhecida como blockchain ${ }^{36}$.

O conceito da blockchain é atualizado e implementado em diversas realidades, por distintas organizações e desenvolvedores. Esta tecnologia pode ser aplicada em diferentes processos de tramitação de registros informacionais. Assim sendo, é possível aplicá-la também na perspectiva da GAD associada ao OAIS. Embora, as técnicas de autenticação por meio de assinatura digital não garantam a autenticidade do documento num longo prazo, pois estão condicionadas à atualização/substituição de hardware, software ou formatos ${ }^{10,37}$. Assim, todos os procedimentos técnicos para a autenticidade dos registros em cadeias de custódias devem ser associados ao modelo referência OAIS ou a outro modelo que atenda a tais requisitos aplicados à realidade das organizações e comunidades envolvidas (i.e. os custodiadores). 


\section{MODELAGEM CONCEITUAL DA CADEIA DE CUSTÓDIA DOS RES: DIRETRIZES PARA OS RDC-ARO EM SAÚDE}

Os repositórios são sistemas disponíveis na web que fornecem facilidades de armazenamento e acesso aos objetos digitais. Os repositórios agregam, ainda, facilidades ao gerenciamento dos objetos digitais neles armazenados (e.g. preservação, recuperação e disseminação dos documentos) ${ }^{38}$. Os repositórios surgem por meio de aplicações da informática e, a partir do século XX, vem sendo desenvolvidos por áreas que investigam a informação (e.g. Ciência da Informação, Biblioteconomia e Arquivologia). A partir de 2010, intensificaram-se os estudos relacionados aos repositórios arquivísticos ${ }^{39}$.

Os repositórios apresentam flexibilidade relacionada às suas finalidades, podendo ser classificados como: institucionais; científicos; produção técnica; temático; arquivístico; monodocumentais, multidocumentais e misto ${ }^{3}$. Assim, a classificação não é única, um repositório pode apresentar mais de uma classificação para atender a sua finalidade ${ }^{39}$. Os repositórios arquivísticos em saúde, objeto desta pesquisa, são os que acumulam informações orgânicas em saúde com a finalidade de disseminá-las.

Os termos disseminação, difusão e divulgação de informações são distintos e os respectivos conceitos são inerentes à modelagem de uma estrutura de RDC-Arq. Na perspectiva das informações orgânicas geradas, recebidas e acumuladas, nos repositórios de saúde, a disseminação é o envio das informações, preferencialmente, pelos postulados da função de disseminação arquivística, para um público especializado (e.g. organismos produtores do SUS, gestores e profissionais de saúde), por meio de técnicas de gestão de documentos. Já a difusão é o envio de mensagens (e.g. dados ou informações) compreensíveis para a totalidade dos agentes de saúde. E a divulgação é a codificação das informações em saúde por meio de linguagens acessíveis para a população.

No Brasil, o RDC-Arq é a referência para implementação de Repositórios Arquivísticos Digitais Confiáveis, portanto ele estabelece as atribuições de um repositório dessa natureza. Entre elas, podemos citar como funções: “(...) gerenciar os documentos e metadados de acordo com as práticas e normas da Arquivologia, especificamente relacionadas à gestão documental, descrição arquivística multinível e preservação; e proteger as características do documento arquivístico, em especial a autenticidade (identidade e integridade) e a relação orgânica entre os documentos. Um repositório digital confiável é um repositório digital que é capaz de manter autênticos os materiais digitais, de preservá-los e prover acesso a eles pelo tempo necessário"19.

É recomendável, para o desenvovlimento dos sistemas ou repositórios de informações orgânicas em saúde, considerar os metadados ou os elementos de descrição da informação, os quais são encontrados nas Normas de Descrição Arquivística, a saber: General International Standard Archival Description (ISAD(G)); International Standard Archival Authority Record for Corporate Bodies, Persons and Families (ISAAR (CPF)); International Standard for Describing Functions (Corporate Bodies, Persons and Families) (ISDF); International Standard for Describing Institutions with Archival Holdings (ISDIAH); Norma Brasileira de Descrição Arquivística (NOBRADE); e o uso do SIGAD (e.g. e-Arq Brasil) para sistemas de informação arquivísticos digitais confiáveis ${ }^{39}$. Um repositório digital de documentos arquivísticos "é um repositório digital que armazena e gerencia (...) documentos, seja nas fases corrente e intermediária, seja na fase permanente"19.

Atualmente, a principal norma recomendada para a preservação digital é o Open Archival Information System (OAIS). O modelo OAIS é estruturado para agrupar dados em pacotes de informações que permitem as funções básicas do repositório, como: submissão, preservação e disseminação. Essas funções básicas são realizadas por meio de três tipos de pacotes de informação: "Pacote de informação para submissão (Submission Information Package - SIP) - refere-se à admissão dos documentos digitais e seus metadados associados. Pacote de informação para arquivamento (Archival Information Package - AIP) - refere-se ao acondicionamento e armazenamento dos documentos digitais e seus metadados associados. Pacote de informação para disseminação (Dissemination Information Package - DIP) - refere-se ao acesso aos documentos digitais e seus metadados associados"19. 
Esses pacotes de informação envolvem os documentos digitais (i.e. informação de conteúdo) e seus metadados (i.e. informação de representação) que são gerenciados por meio de grupos desenvolvidos pela TRAC, a saber: Admissão - captura de documentos digitais e criação do pacote de arquivamento; Planejamento da preservação; Armazenamento e preservação/ manutenção do AIP; Gerenciamento de informação; Gerenciamento de acesso ${ }^{19}$. O OAIS deve ser implantado entre os sistemas integrantes da cadeia de custódia dos documentos digitais para que ocorra a circulação dos pacotes SIP, AIP e DIP. Por meio de protocolos padronizados são realizadas solicitações por sistemas designados que respondem com um envelope que contém um registro de metadados identificados e criptografados, de acordo com o documento associado ao registro em conformidade com um formato especificado na solicitação. Para cada grupo existem requisitos a serem cumpridos que estão descritos nos RDC-Arq ${ }^{19}$.

Existem softwares livres voltados para a gestão de repositórios dessa natureza que “(...) não estão exatamente sob uma implementação de política de preservação digital, já que os documentos contidos na base da ferramenta são representantes digitais" ${ }^{\circ}$, sendo assim, faz-se necessária a sua integração com outros sistemas". Os repositórios, na atualidade, podem utilizar o SIGAD associado ao Archivematica e vinculados ao AtoM (Figura 1).

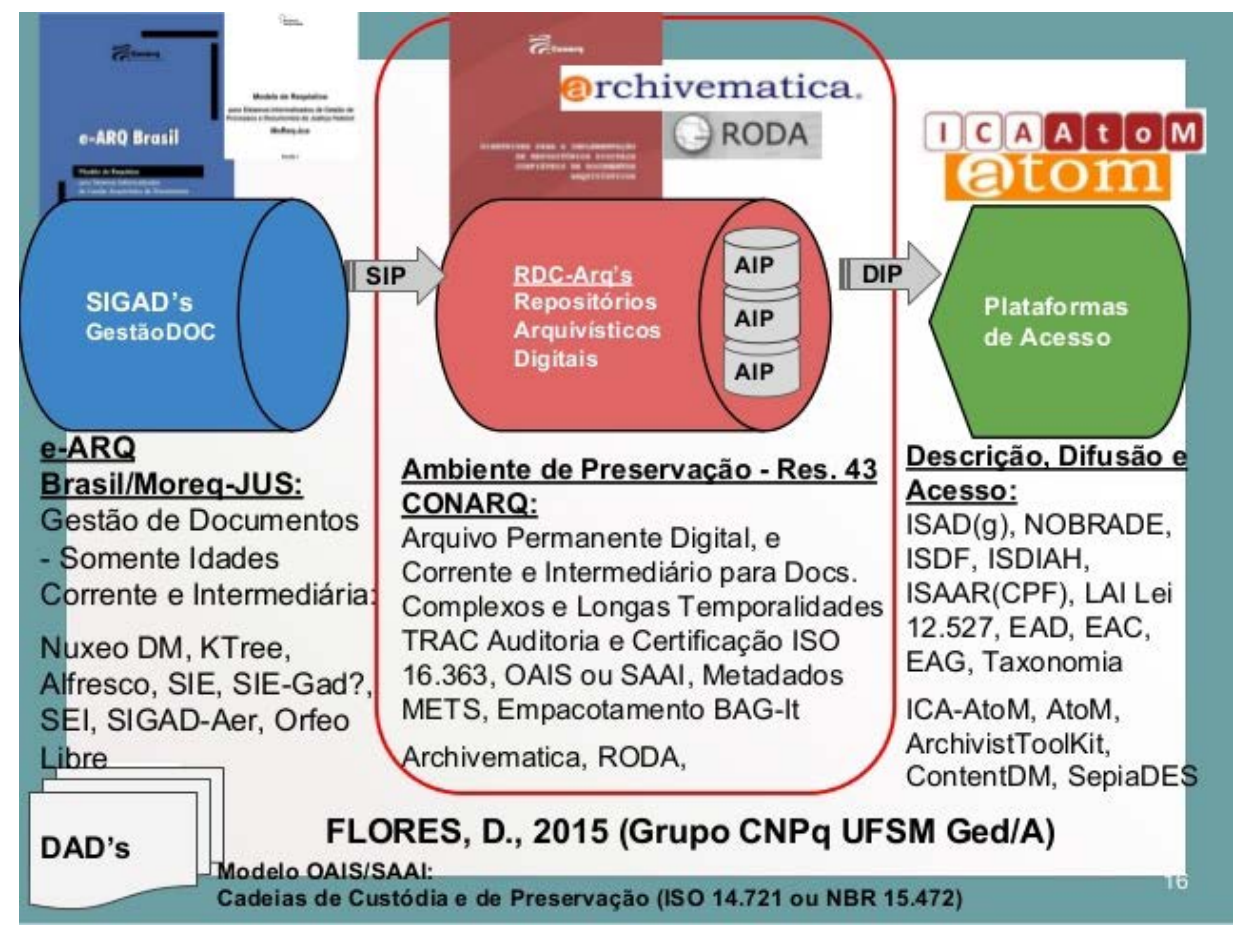

Figura 1 - Cadeia de preservação por meio de softwares especializados Fonte: Flores (2017).

É recomendável que os documentos arquivísticos digitais, em fase corrente e intermediária, sejam gerenciados por um SIGAD. Faz-se necessário que os SIGAD sejam interoperáveis com sistemas que estejam em conformidade com políticas de preservação digital, normas e modelos como o ISO-OAIS, por exemplo, o Archivematica - software voltado para preservação de documentos arquivísticos digitais -, e com sistemas voltados para a descrição arquivística e que primem pela guarda permanente e difusão, a exemplo do AtoM. Os repositórios, assim desenvolvidos, contemplam todas as idades documentais e possibilitam a preservação, autenticidade e a organicidade dos documentos.

No âmbito do SUS, o DATASUS, é considerado o repositório das informações do SUS e os principais SIS são "os de mortalidade (SIM), de nascimento (SINASC), ambulatorial (SIA-SUS), de internações hospitalares (SIH), de notificações de doenças (SINAN), de atenção básica (SIAB), [...] para níveis populacionais”3. 
No entanto, não fica evidente se as informações recebidas, acumuladas e difundidas por meio do DATASUS ou mesmo se as informações geradas, recebidas, acumuladas e disseminadas pelos OPSAS para o DATASUS utilizam métodos e técnicas da Arquivologia para a autenticidade e a preservação dos RES.

Pesquisas realizadas ${ }^{41-43}$ revelam a ausência de procedimentos de gestão arquivística de documentos nos OPSAS da amostra e de profissionais qualificados para o tratamento e organização dos documentos. Revelam ainda um desconhecimento por parte dos gestores dos setores de TI e de Arquivos acerca da importância da gestão e da avaliação das informações orgânicas em saúde e de como é realizada a padronização e a recuperação das informações em relação ao DATASUS ${ }^{42,43}$. A ausência da GAD e de profissionais qualificados em relação às técnicas e aos procedimentos arquivísticos compromete o fluxo de informações entre os OPSAS e o DATASUS e, consequentemente, a cadeia de custódia do SUS.

A partir dos resultados encontrados e por meio da revisão de literatura e documental foi possível identificar os custodiadores em saúde (e.g. OPSAS e Portal do DATASUS) e sistematizá-los em uma proposta de modelagem conceitual da cadeia de custódia para os RES do SUS (Figura 2).

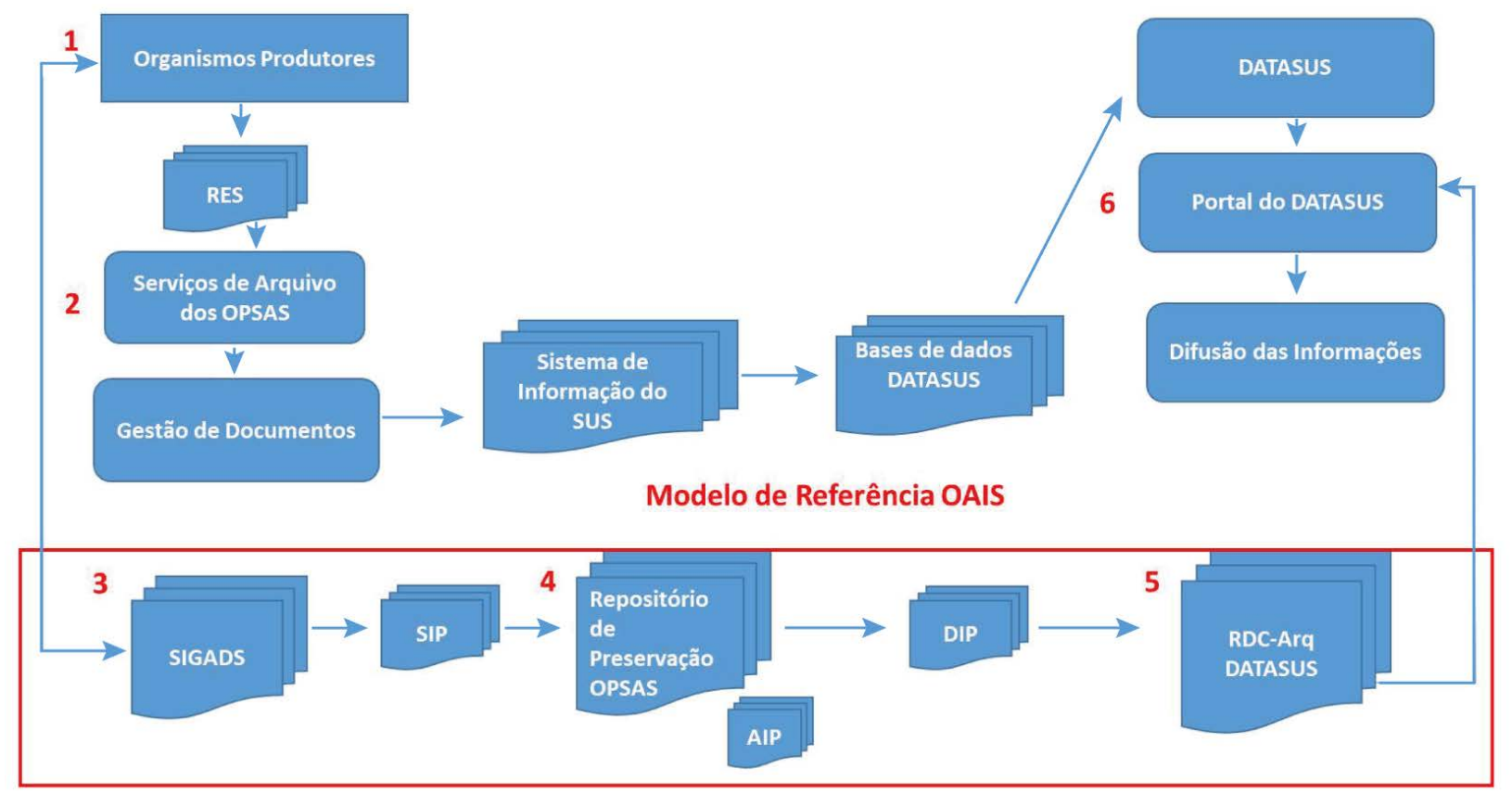

Figura 2 - Proposta de modelagem conceitual da cadeia de custódia do SUS Fonte: Os autores (2020).

Na Figura 2 é apresentado o fluxo das informações dos OPSAS (e.g. Atenção Primária à Saúde, atenção especializada e atenção de alta complexidade) para o DATASUS. A Figura 2 ilustra uma proposta de uma modelagem para a garantia da autenticidade e preservação dos RES e da cadeia de custódia do SUS por meio do OAIS. As etapas deste fluxo são descritas a seguir:

Organismos Produtores (e.g. Atenção Primária à Saúde, atenção especializada e atenção de alta complexidade). Nos OPSAS são produzidas informações orgânicas em saúde. Essas informações são registradas nos PP e em outras tipologias documentais que, em formato eletrônico, conformam os RES, produzidos e utilizados pelas áreas assistencial e administrativa diariamente. Na perspectiva deste trabalho, os produtores modelados foram os OPSAS (com termo de adesão a uma rede de inovação e aprendizagem em gestão hospitalar) e o Portal do DATASUS. O uso da GAD desde a gênese documental é imprescindível para que as informações geradas não percam a autenticidade por falta de tratamento qualificado;

Os Serviços de Arquivo (SA) são responsáveis por gerir os documentos que subsidiam os profissionais e os gestores de saúde nos processos de tomada de decisão. É recomendado o uso da GAD que engloba as 
técnicas de tratamento e a organização dos RES por meio dos SA, em consonância com o desenvolvimento dos SIS (TI/Computação), como também a atuação do arquivista, profissional especializado em GAD;

Os dados imputados nos SIS dos OPSAS devem ser gerenciados por meio de SIGAD para que sejam interoperáveis com sistemas de preservação digital, em conformidade com o modelo ISO-OAIS. O OAIS deve ser implantado entre os sistemas integrantes da cadeia de custódia dos documentos digitais para que ocorra a circulação dos pacotes SIP, AIP e DIP, de modo que a cadeia possa seguir garantindo a sua autenticidade e preservação ininterrupta;

Os RES armazenados nos SIGAD dos OPSAS devem ser submetidos, por meio do SIP, e arquivados pelo AIP, nos repositórios de preservação dos OPSAS. Os dados solicitados pelo MS aos OPSAS serão enviados via DIP para os repositórios do DATASUS, seguindo o mesmo fluxo da cadeia de custódia, de acordo com o modelo referência OAIS até serem enviados para o Portal do DATASUS.

Os repositórios do DATASUS necessitam ter características de RDC-Arq em razão da relevância das informações orgânicas em saúde para garantir a autenticidade e preservação dos RES.

As informações geradas pelos OPSAS e tratadas pelo DATASUS são disponibilizadas no Portal do DATASUS, que possibilita o acesso e a recuperação das informações em saúde. Essas informações necessitam de mecanismos de Difusão/Disseminação/Divulgação para atender às distintas necessidades dos profissionais e gestores de saúde e da população em geral, com a garantia da autenticidade das informações disponibilizadas.

A modelagem conceitual proposta de uma cadeia de custódia do SUS visa potencializar a operacionalização das RAS, uma vez que este sistema de saúde "[...] representa uma ruptura no formato de constituição do Estado, no modelo de proteção social e na forma de gestão das políticas sociais no país, tendo, portanto, redirecionado também o padrão de relacionamento do Estado com a sociedade civil e o mercado. Este processo gerou arranjos multiorganizacionais, característicos de governança em rede, onde a capacidade de desenvolver estratégias e instrumentos de promoção e sustentação de interdependências se tornou vital para o desempenho final do sistema de saúde. Isto é, envolve o planejamento e a articulação estratégica no emprego dos recursos, a negociação em torno de objetivos comuns, a pactuação de metas, a resolução mediada de conflitos, a integração por meio da articulação eficiente de bases de provisão de serviços, entre outros" 44

Espera-se que os custodiadores do SUS modelados, na Figura 2, assimilem subsídios para a criação de repositórios digitais em saúde confiáveis e recomenda-se a adoção e a incorporação dos processos da GAD articulados com os da TI. Tal assimilação subsidia a geração de conhecimentos em saúde para as intervenções administrativas e assistências no âmbito do SUS.

\section{CONSIDERAÇÕES SOBRE UMA MODELAGEM DA CADEIA DE CUSTÓDIA DOS RES PARA O SUS}

A revisão sistemática realizada para prospecção e análise sobre autenticidade e preservação dos RES e da cadeia de custódia do SUS subsidiou a identificação dos custodiadores, sistematizados para a modelagem conceitual do fenômeno (i.e. a infraestrutura de sistemas de gestão da preservação e Repositórios Digitais Confiáveis ó apresentando os agentes custodiadores dos RES no âmbito das RAS do SUS) entre os OPSAS e o Portal do DATASUS, os quais geram, recebem e acumulam documentos arquivísticos diariamente.

Espera-se que a revisão apresentada e a proposta de modelagem conceitual da cadeia de custódia para informações do SUS, entre os OPSAS e o DATASUS, tragam subsídios para criação de Repositórios Digitais Confiáveis em saúde. É recomendável a adoção e a incorporação do processo de gestão e preservação documental em Repositórios Digitais Confiáveis para um efetivo Sistema Nacional de Informação em Saúde (SNIS). 
Está sendo planejada uma simulação no laboratório do Grupo de Estudos de Políticas de Informação, Comunicações e Conhecimento (GEPICC), no qual essa investigação está vinculada, com o fluxo de tramitação da produção dos RES, de um dos OPSAS da amostra, até o envio ao Portal do DATASUS e, posteriormente, sua aplicação no modelo OAIS. Pretende-se testar os softwares Archivists' Toolkit associados ao Archivematica e vinculados ao AtoM, entre outros, para exemplificar o funcionamento da cadeia de custódia com base no OAIS, cruzando com os resultados encontrados nas entrevistas dos gestores dos OPSAS e do DATASUS, para a preservação da cadeia de custódia e a difusão das informações orgânicas em saúde por meio do Portal do DATASUS.

Estudos voltados à assimilação de Repositórios Digitais Confiáveis nos serviços de atenção à saúde e nas três esferas de Governo, voltados à autenticidade, preservação e manutenção da cadeia de custódia das informações em saúde, são necessários para o Brasil. Visto que ainda existem muitos obstáculos entre a adoção e a assimilação e entre os sujeitos e as organizações, respectivamente, de tecnologias avançadas de produção, disseminação e acesso às informações gerenciais de serviços de atenção à saúde (e.g. GAD, sistemas de arquivos e de repositórios arquivísticos). Contudo, com o enfrentamento da pandemia de Covid-19, as desigualdades socioeconômicas no Brasil e as dificuldades para propor soluções de ordem econômica e sanitária ficam ainda mais evidentes. É um cenário sobre o qual ainda não é possível fazer previsões, mas que será de recessão para todos os setores da economia e, consequentemente, para os serviços de arquivo, que possuem investimentos limitados.

Esta proposta de modelagem contribui com as estratégias de gestão objetivando o fortalecimento do SUS; e, “(...) mais do que trazer certezas, objetiva oferecer bases para ampliar o debate sobre as condições de possibilidade para tornar concreto o direito universal à saúde" 45 . Para finalizar questionamos: de que forma os SIS podem se adequar às demandas das distintas territorialidades do país? Como garantir a preservação e a organização das informações geradas, recebidas e acumuladas nos repositórios em saúde? Como será a rotina dos profissionais envolvidos para o alcance de uma modelagem dessa natureza? Quais competências os gestores e profissionais da saúde das RAS devem adquirir para efetivar a modelagem proposta?

As respostas para essas questões merecem ser ponderadas, refletidas e equacionadas, considerandose as perspectivas socioeconômicas e culturais dos agentes institucionais do SUS. Associadas, ainda, às realidades das distintas territorialidades do Brasil e, consequentemente, dos OPSAS, com a participação da sociedade civil e dos profissionais envolvidos nos contextos da atenção à saúde, sem os quais nenhum modelo funcionará com efetividade.

\section{REFERÊNCIAS}

1. Formenton D, Castro FF, Gracioso LS, Furnival ACM, Simões MGM. Os padrões de metadados como recursos tecnológicos para a garantia da preservação digital. Biblios: Journal of Librarianship and Information Science [Internet]. 2017 jan. [citado em 2020 abr. 20];68:82-95. Disponível em: https:// biblios.pitt.edu/ojs/index. php/biblios/article/view/414.

2. Ministério da Saúde (BR). Política Nacional de Informação e Informática em Saúde [Internet]. Brasília, DF: Ministério da Saúde; 2016 [citado em 2019 dez. 5]. Disponível em: http://bvsms.saude.gov.br/bvs/ publicacoes/politica nacional infor informatica saude 2016.pdf.

3. Pinto LF, Freitas MPS, Figueiredo AWS. Sistemas Nacionais de Informação e levantamentos populacionais: algumas contribuições do Ministério da Saúde e do IBGE para a análise das capitais brasileiras nos últimos 30 anos. Ciênc. saúde coletiva [Internet]. 2018 jun. [citado em 2020 maio 18]; 23(6):1859-1870. p. 1860. Disponível em: http://www.scielo.br/ scielo.php?script=sci arttext\&pid=S1413-81232018000601859\&lng=en.

4. Pinto VB, Soares ME. Apresentação. In: Pinto VB, Soares ME. Informação para a área de saúde. Fortaleza: Edições UFC; 2010. p. 13-22. 
5. Cunha FJAP, Lima GLQ, Oliveira LAF. Arquivos como mecanismos de difusão de conhecimentos para a aprendizagem e inovação em organismos produtores de saúde. In: Anais da $4^{a}$ Reunião Brasileira de Ensino e Pesquisa em Arquivologia, 4. 2015 ago. 3-6. João Pessoa: UFPB; 2015.

6. Cunha FJAP. O complexus do conhecimento, inovação e comunicação em serviços de atenção à saúde. In: Cunha FJAP, Lázaro CP, Pereira HBB., organizadores. Conhecimento, inovação e comunicação em serviços de saúde. Salvador: EDUFBA; Rio de Janeiro: Fiocruz; 2014b. p. 221-236.

7. Sistema Único de Saúde (BR). Departamento de Informática do SUS [Internet]. Brasília, DF: Ministério da Saúde; 2020a [citado em 2020 maio 5]. Disponível em: http://www2.datasus. gov.br/DATASUS/ index.php.

8. Associação Brasileira de Pós-Graduação em Saúde Coletiva. Grupo Temático Informação em Saúde e População da Abrasco. $2^{\circ}$ Plano Diretor de Desenvolvimento da Informação e Tecnologia de Informação em Saúde 2013-2017 [Internet]. Rio de Janeiro: ABRASCO; 2015 [citado em 2020 maio 5]. Disponível em: http://www.abrasco.org.br/ckfinder/userfiles/files/PlaDITIS\%202013-17\%202a\%20Versao\%20 Consulta\%20publ\%20(1).pdf.

9. Sistema Único de Saúde (BR). Estratégia e-Saúde para o BRASIL [Internet]. 2014 [citado em 2019 dez. 5]. Disponível em: http://cspace.eportuguese.org/tiki-download file.php?fileId=1173.

10. Conselho Nacional de Arquivos (BR). Câmara Técnica de documentos eletrônicos. Diretrizes para a presunção de autenticidade de documentos arquivísticos digitais. 2012 [citado em 2020 mar. 3]. Disponível em: http://www.conarq.arquivonacional.gov.br/media/Diretrizes pre suncao autenticidade\%20finalizada.doc.

11. Lousada M, Valentim MLP. A relação entre a informação orgânica e a gestão documental [Internet]. In: Valentim MLP., organizadora. Gestão, mediação e uso da informação. São Paulo: Editora UNESP; São Paulo: Cultura Acadêmica; 2010 [citado em 2019 mar. 1]. Disponível em: http://books.scielo.org/id/ j4gkh/pdf/valentim-9788579831171-18.pdf.

12. Sousa MR, Ribeiro ALP. Revisão Sistemática e meta-análise de estudos de diagnóstico e prognóstico: um tutorial. Arq. Bras. Cardiol. [Internet]. 2009 mar. [citado em 2020 maio 5];92(3):241-251. Disponível em: http://www.scielo.br/scielo.php?script=sci arttext\&pid=S0066-782X2009000300013.

13. Sampaio RF, Mancini MC. Estudos de Revisão Sistemática: um guia para síntese criteriosa da evidência científica. Rev. Bras. Fisioter. [Internet]. 2007 jan.fev. [citado em 2020 maio 5];11(1):83-89. Disponível em: http://www.scielo.br/scielo.php?script=sci arttext\&pid=S1413-35552007000100013.

14. Varnienè-Janssen R, Kuprienè J. Autentiškumas ir proveniencija ilgalaikio skaitmeninio išsaugojimo kontekste: turinio aprèpties analizè. Informacijos mokslai [Internet]. 2018 [citado em 2020 abr. 25];82:131-160. Disponível em: https://epublications.vu.It/object/elaba: 33983103/index.html.

15. Santos HM, Flores D. Novos rumos da preservação digital: das estratégias aos sistemas informatizados. Biblios: Journal of Librarianship and Information Science [Internet]. 2018 fev. [citado em 2020 abr. 25];70:31-43. Disponível em: https://biblios.pitt.edu/ojs/index.php/biblios/article/view/326.

16. Sayão LF. Uma outra face dos metadados: informações para a gestão da preservação digital. R. Eletr. Bibliotecon. Ci. Inf. [Internet]. 2010 [2015 fev 10];15(30):1-31. Disponível em: https://periodicos.ufsc. br/index.php/eb/article/view/1518-2924.2010v15n30p1.

17. Bountouri L, Gratz P, Sanmartin F. Digital Preservation: How to Be Trustworthy. In: Ioannides M, editor. Digital Cultural Heritage. Cham: Springer; 2018.

18. Conselho Nacional de Arquivos (BR). Câmara Técnica de documentos eletrônicos. Carta para a Preservação do Patrimônio Arquivístico Digital [Internet]. Rio de Janeiro: Arquivo Nacional; 2004 [citado em 2020 maio 5]. Disponível em: http://conarq.gov.br/images/publicacoes textos/Carta preservacao. pdf.

19. Conselho Nacional de Arquivos (BR). Câmara Técnica de documentos eletrônicos. Diretrizes para a Implementação de Repositórios Arquivísticos Digitais Confiáveis - RDC-Arq [Internet]. Rio de Janeiro: Arquivo Nacional, 2015 [citado em 2020 mar. 30]. Disponível em: http://www.conarq.arquivonacional. gov.br/media/publicacoes/resol conarq 39 repositorios.pdf.

20. Barros DBS, Ferrer ID, Maia CMS. Auditoria de repositórios digitais preserváveis. RICI: R.Ibero-amer. Ci. Inf. [Internet]. 2018 [citado em 2020 maio 5];11(1):300-31. Disponível em: https://periodicos.unb.br/ index.php/RICI/article/view/8572.

21. Cunha FJAP, Lima GLQ, Oliveira LAF, Meirelles RF. Manual de gestão de documentos arquivísticos em saúde. Salvador: EDUFBA; 2020. (No prelo). 
22. Sistema Único de Saúde (BR). Departamento de Informática do SUS (DATASUS). Plano de Contingência DATASUS situação de crise provocada pelo Novo Coronavírus (Covid-19) [Internet]. Brasília, DF: Ministério da Saúde; 2020b [citado em 2020 maio 5]. Disponível em: https://datasus.saude.gov.br/wpcontent/uploads/2020/04/Plano-de-conting\%C3\%AAncia-DATASUS.pdf.

23. International Research on Permanent Authentic Records in Electronic Systems Interpares 2 Project. Diretrizes do Preservador: a preservação de documentos arquivísticos digitais: diretrizes para organizações [Internet]. 2017 [citado em 2020 abr. 25]. Disponível em: https://www.siarq. unicamp.br/ siarq/images/siarq/pesquisa/pdf/diretrizes preservador.pdf.

24. Wilson T. Rethinking digital preservation: definitions, models, and requirements. Digital Library Perspectives [Internet]. 2017 [citado em 2020 maio 5];33(2):128-136. doi: https://doi.org/10.1108/ DLP-08-2016-0029.

25. Térmens M, Leija D. Auditoría de preservación digital con NDSA Levels, El Profesional de la Información [Internet]. 2017 [citado em 2020 maio 5];26(3):447-456. Disponível em: https://www.scipedia.com/ public/Termens Leija 2017a.

26. Constituição 1988 (BR). Constituição da República Federativa do Brasil [Internet]. Brasília, DF: Senado Federal; 1988 [citado em 2019 dez. 5]. Disponível em: http://www.senado.gov.br/ atividade/const/ con1988/CON1988 05.10.1988/art 5 .asp.

27. Lei no 13.709 (BR), 14 de agosto de 2018. Lei Geral de Proteção de Dados Pessoais. DOU 2018 ago 15 [citado em 2020 mar. 5]. Disponível em: http://www.planalto.gov.br/ccivil 03/ ato2015-2018/2018/lei/ 113709.htm.

28. Controladoria Geral da União (BR). Acesso à Informação Pública. Uma introdução à Lei no 12.527 , de 18 de novembro de 2011. Brasília, DF: CGU; 2011 [citado em 2019 dez. 5]. Disponível em: https://www. gov.br/acessoainformacao/pt-br/central-de-conteudo/publicacoes/arquivos/cartilhaacessoainformacao-1. pdf.

29. Conselho Federal de Medicina (Brasil). Resolução CFM no 1931, de 17 de setembro de 2009. Contém as normas éticas que devem ser seguidas pelos médicos no exercício da profissão, independentemente da função ou cargo que ocupem [Internet]. DOU 2009 out. 13 [citado em 2020 mar. 5]. Disponível em: http://portal.cfm.org.br/index.php?option=com content\&view=article\&id=20670:resolucao-cfm-no19312009-\&catid=9: codigo-de-etica-medica-atual\&Itemid=122.

30. Galeffi DA. Ética, privacidade e confidencialidade de informação em saúde. Inf. Pauta [Internet]. 2020 mar. [citado em 2020 maio 18];5(Especial 1):9-22. Disponível em: http://www.periodicos.ufc.br/ informacaoempauta/article/view/43509.

31. Suderman J. Principle-based concepts for the long-term preservation of digital records [Internet]. In: Proceedings of the 1st International Digital Preservation Interoperability Framework Symposium; 2010 Apr; New York: [ACM]; 2010 [citado em 2020 set 2]. Disponível em: https://dl.acm.org/doi/ abs/10.1145/2039263.2039270.

32. Flores D. Documentos Arquivísticos Digitais: gestão, preservação e acesso [slide]. Porto Alegre; 2017 [citado em 2020 abr. 20]. Slides: color, 4x3. Material elaborado para Palestra na Assembleia Legislativa do Estado do Rio Grande do Sul, no I Seminário de Preservação Digital, ocorrido em 07 de agosto de 2017. Disponível em: http://documentosdigitais.blogspot.com.

33. Instituto Nacional de Tecnologia da Informação (BR). Infraestrutura de Chaves Públicas Brasileira - ICPBrasil [Internet]. Brasília, DF: Casa Civil da Presidência da República; 2017 [citado em 2020 maio 5]. Disponível em: https://www.iti.gov.br/icp-brasil.

34. Ministério da Saúde (BR). Secretaria de Atenção Primária à Saúde. e-SUS Atenção Básica: Manual do Sistema com Prontuário Eletrônico do Cidadão PEC - Versão 3. Brasília, DF: Ministério da Saúde; 2019 [citado em 2020 mar. 5]. Disponível em: http://aps.saude.gov.br/ape/esus/manual 3 2/introdutorio.

35. Conselho Federal de Medicina (BR). Resolução CFM no 1.638 de 10 de julho 2002. Define prontuário médico e torna obrigatória a criação da Comissão de Revisão de Prontuários nas Instituições de Saúde [Internet]. DO 2002 ago 9 [citado em 2020 mar. 5]. Disponível em: http://www.portalmedico.org.br/ resolucoes/cfm/2002/1638 2002.htm.

36. Nakamoto S. Bitcoin: A peer-to-peer electronic cash system [Internet]. 2008 [citado em 2020 maio 5]. Disponível em: https://bitcoin.org/bitcoin.pdf. 
37. Schuch, CDOS, Saad DS, Flores D. Preservação digital na gestão de processos administrativos de uma instituição de ensino superior: o caso dos PEAPDs da PROGEP da UFSM. Em Questão [Internet]. 2019 maio-ago. [citado em 2020 abr. 25];25(2):229-255. Disponível em: https://seer.ufrgs.br/EmQuestao/ article/view/82597.

38. Shintaku M, Meirelles RF. Manual do DSPACE: administração de repositórios [Internet]. Salvador: Edufba, 2010 [citado em 2020 abr. 25]. Disponível em: https://repositorio.ufba.br/ri/handle/ri/769.

39. Meirelles RF, Cunha FJAP. Repositórios Arquivísticos Digitais. In: Galeffi DA, Marques MIC, Santos CAS, Rocha-Ramos M., organizadores. Transciclopédia termos e usos em difusão do conhecimento: criando conceitos, fuctivos, perceptos/ afetos e intuitos. Salvador: Quarteto Editora; 2020. No prelo.

40. Flores D. Os Repositórios Arquivísticos Digitais. Ci. Inf. 2013 jan.-abr.;41(1):81-97.

41. Cunha FJAP, Lima GLQ, Oliveira LAF, Meirelles RF. Informação, documentos, arquivos e repositórios: mecanismos de difusão de conhecimentos para as inovaçõ̃es gerenciais nos sistemas de saúde. In: Barros THB, Santos Junior RL dos, Cândido GG., organizadores. A pesquisa e o ensino em arquivologia: perspectivas na era digital. Belém: Ed. da UFPA; 2019. (v. 1).

42. Lima GLQ. As formações discursivas da gestão arquivística e a difusão do conhecimento nos OPSAS: uma análise a partir das práticas de tratamento e organização das informações orgânicas em saúde [tese]. Salvador: Universidade Federal da Bahia; 2018 [citado em 2020 mar]. 189 p. Disponível em: https://repositorio.ufba.br/ri/handle/ri/28764.

43. Souza ACC. Informação e tecnologias de informação em saúde: fontes e mecanismos de transferência de conhecimento para a gestão do SUS em hospitais com termo de adesão à Rede INOVARH-BA 2017 [dissertação]. Salvador: Universidade Federal da Bahia; 2017 [citado em 2019 mar. 5]. 146 p. Disponível em: https://repositorio.ufba.br/ri/bitstream/ri/22998/1/Angela\% 20Cristina\%20Cordeiro\%20-020 dissertacao.pdf.

44. Fleury S, Ouverney A. O Sistema Único de Saúde brasileiro: desafios da gestão em rede. Rev. Portuguesa e Brasileira de Gestão [Internet]. 2012 [citado em 2020 maio 18];11(2-3):74-83. Disponível em: http://www.scielo.mec.pt/scielo.php?pid=S164544642012000200007 \&script=sci abstract.

45. Campos GWS. SUS: o que e como fazer? Ciênc. saúde coletiva [Internet]. 2018 jun. [citado em 2020 maio 18];23(6):1707-1714. Disponível em: http://www.scielo.br/scielo.php?script=sci arttext\&pid=S1413-81232018000601707\&lng=en. 